\title{
Synchrony and Elementary Operations on Coupled Cell Networks
}

\author{
M.A.D. Aguiar ${ }^{1}$, A.P.S. Dias ${ }^{2}$ and H. Ruan ${ }^{3, *}$ \\ ${ }^{1}$ Centro de Matemática da Universidade do Porto, \\ Rua do Campo Alegre, 687, 4169-007 Porto, Portugal \\ Faculdade de Economia, Universidade do Porto, \\ Rua Dr Roberto Frias, 4200-464 Porto, Portugal \\ 2 Departamento de Matemática, Centro de Matemática, Universidade do Porto, \\ Rua do Campo Alegre, 687, 4169-007 Porto, Portugal \\ 3 Fachbereich Mathematik, Universität Hamburg, \\ Bundesstraße 55, 20146 Hamburg, Germany
}

E-mail: maguiar@fep.up.pt apdias@fc.up.pt haibo.ruan@math.uni-hamburg.de

July 22, 2015

\begin{abstract}
Given a finite graph (network), let every node (cell) represent an individual dynamics given by a system of ordinary differential equations, and every arrow (edge) encode the dynamical influence of the tail node on the head node. We then have defined a coupled cell system that is associated with the given network structure. Subspaces that are defined by equalities of cell coordinates and left invariant under every coupled cell system respecting the network structure are called synchrony subspaces. They are completely determined by the network structure and form a complete lattice under set-inclusions. We analyze the transition of the lattice of synchrony subspaces of a network that is caused by structural changes in the network topology, such as deletion and addition of cells or edges, and rewirings of edges. We give necessary, and in some cases sufficient, conditions under which lattice elements persist or disappear.
\end{abstract}

AMS classification scheme numbers: 34C15 37C10 05C76 06B23

Keywords: Coupled cell network; graph operation; coupled cell system; synchrony subspace; lattice.

\footnotetext{
${ }^{*}$ Research in part supported by the Deutsche Forschungsgemeinschaft under grant DFG RU 1748/2-1.

${ }^{+}$Research funded by the European Regional Development Fund through the programme COMPETE and by the Portuguese Government through the FCT - Fundação para a Ciência e a Tecnologia under the project PEst-C/MAT/UI0144/2011 and PTDC/MAT/100055/2008.
} 


\section{Introduction}

In the theory of coupled cell networks developed by Stewart, Golubitsky and their co-workers in [15, 10,9] or Field in [8], a network is a finite set of nodes (or cells) linked together by a finite number of arrows, and dynamical systems that are consistent with this network structure are called the admissible coupled cell systems. More precisely, every cell of the network represents an individual dynamics given by a finite set of ordinary differential equations and each arrow describes the interaction between the two connected individuals. In analogue to other structures of dynamical systems such as symmetry or the Hamiltonian, network structure imposes strong restrictions on the dynamics of the associated coupled cell systems. One striking example is the existence of synchrony subspaces, which are spaces defined by equalities of cell coordinates that are flow-invariant for all coupled cell systems associated with the given network structure. Coupled cell systems restricted to such synchrony subspaces are again coupled cell systems associated with smaller networks, called the quotient networks, and characterize the dynamics of the initial coupled cell systems subject to the equalities defining the specific synchrony subspace.

Synchrony subspaces contribute to the dynamics of the coupled cell systems through these restricted systems. See, for example, the paper by Aguiar et al. [1] where coupled cell systems supporting heteroclinic behavior are analyzed and part of the crucial elements guaranteeing that kind of dynamics is the existence of synchrony subspaces for which the restricted systems have the desired dynamic properties.

Synchrony subspaces can be determined solely by the underlying network structure. More precisely, Stewart, Golubitsky and their co-workers proved in $[15,10]$ that synchrony subspaces of coupled cell systems associated with a network structure are in one-to-one correspondence to those equivalence relations among the cells of the network that satisfy a combinatorial property, called balanced.

By Stewart [14], the set of all synchrony subspaces of a network forms a complete lattice under the set-inclusion; or equivalently, the set of all balanced equivalence relations on the network is a complete lattice under the refinement of equivalence relations (see also Aldis [5]).

Aguiar and Dias [2] showed how to obtain the lattice of synchrony subspaces for a general network and presented an algorithm that generates this lattice. Indeed, it is proved that this problem can be reduced to finding the lattice of synchrony subspaces for networks with only one cell type and one edge type.

In the realm of networks in nature and science, it is a common knowledge that networks having different network topology support different patterns of dynamic behavior. A vivid example is the gene regulatory networks, which, under different conditions, exhibit different regulation patterns accompanied by different transcriptional network topologies (cf. Zhang et al. [16] and Luscombe et al. [13]). As one of the most investigated network-specific dynamic, network synchronizability shows to vary as the network structure varies. There is a vast literature on how synchronizability varies with the structure using different definitions of synchronizability and by varying different network structural properties. Closely related with the graph operations considered in this work, we mention some specific references. Atay and Biyikoglu [6], 
analyzed the effect of graph operations on the synchronization of coupled dynamical systems. The graph operations considered range from unary operations, such as addition or deletion of edges, to binary operations, such as product, join and coalescence. The work of Chen and Duan [7] introduced new results on the synchronizability of coalescences and a condition under which a network and its complementary network have the same synchronizability. In [12], Lu et al. studied the local complete synchronization of discrete-time dynamical networks with time-varying couplings. Furthermore, Hagberg and Schult [11] discussed how to engineer a diffusively coupled network using elementary edge operations to enhance the network synchronization.

As mentioned earlier, in the context of coupled cell systems, the connecting topology of a network dictates the lattice of balanced equivalence relations (or equivalently, the lattice of synchrony subspaces). Thus, it is natural and of interest to ask how these lattices evolve as the underlying topology of the network changes. In this perspective, Aguiar and Ruan [4] considered non-product binary operations on networks such as the join and the coalescence; Aguiar and Dias [3] addressed the same question for product operations on networks.

In this paper we evaluate the impact, on the lattice of balanced equivalence relations of a network, of structural changes in the network topology through elementary graph operations such as addition and/or deletion of cells and/or edges, and/or rewiring of edges. We consider networks with only one cell type and one edge type. Let $\mathcal{G}_{2}$ be a network obtained from a network $\mathcal{G}_{1}$ by an elementary graph operation on edges or nodes.

As one can expect, the lattice of balanced equivalence relations changes in general. We describe necessary, and in some cases sufficient, conditions for balanced equivalence relations to persist or disappear. The information on which balanced equivalence relations remain have interest from the point of view of the real life applications. Consider, for example, an economic framework where agents, who are located at the nodes of a network, need to decide constantly how their relation connections should evolve. The information on the balanced relations that are inherited by the new economic relation network is useful if the agents intend to modify their links over time depending on whether they want to keep synchronizing or not with certain agents in the network.

The paper is organized as follows. In Section 2, we introduce notations and give a compact presentation about coupled cell networks and synchrony subspaces. Our main results appear in Sections 3-4. In Section 3.1 we analyze the effect of removing and/or adding edges upon the lattice of balanced equivalence relations of the network. The main result is Theorem 3.1 where it is shown that an equivalence relation is simultaneously balanced on two networks related by deletion and addition of edges if and only if it is balanced for the two networks, $G_{F}$ and $G_{E}$, having the same set of cells as the two original networks but only with edges corresponding, one to the deleted edges and the other one to the added edges, respectively. This result can be very useful from the applications point of view. Consider, for example, the situation where a big network has a certain synchrony subspace, where the dynamics of the network equations restricted to that synchrony subspace is relevant for the problem being studied - for example, a synchrony-breaking steady-state or Hopf bifurcation. Suppose that 


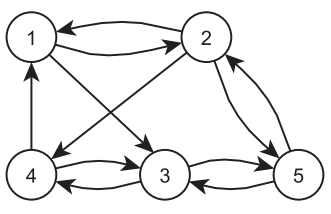

$G_{1}$

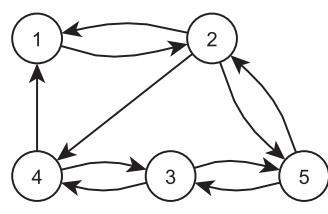

$G_{2}$

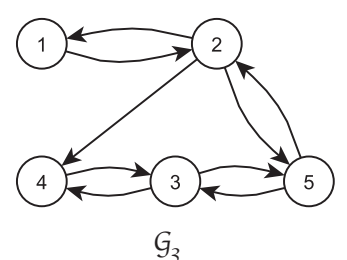

$G_{3}$

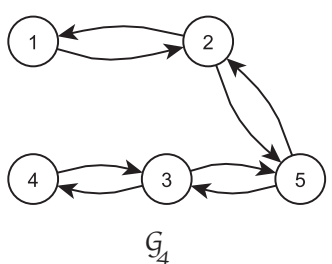

Figure 1: Three networks $\mathcal{G}_{1}, \mathcal{G}_{2}, \mathcal{G}_{3}$, where $\mathcal{G}_{2}$ is obtained from $\mathcal{G}_{1}$ by removing the edge $1 \rightarrow 3$ and $\mathcal{G}_{3}$ is obtained from $\mathcal{G}_{2}$ by removing the edge $4 \rightarrow 1$.

it is necessary to investigate if, under some changes at the network topology, such as edges deletion and/or addition, that dynamics is preserved. A necessary condition for that to happen is the preservation of that synchrony subspace. To check that, using our results, it reduces to check if two very simple networks, $G_{F}$ and $G_{E}$, have that synchrony subspace. Note that, in principle, these two networks have very few edges and so, the problem is now reduced to a much simpler one. Besides Theorem 3.1, further practical result can be inferred using the input equivalence relation. Two cells are called input equivalent, if they receive the same number of input edges for each edge type. Moreover, any balanced equivalence relation must be a refinement of the input equivalence relation on the network. It thus follows from Corollary 3.2 that if a network $\mathcal{G}_{2}$ is obtained from $\mathcal{G}_{1}$ by deleting a single edge $d \rightarrow c$ where $c$ is not input equivalent to any other cell on $\mathcal{G}_{2}$, then the balanced equivalence relations on $\mathcal{G}_{2}$ are precisely the balanced equivalence relations $\bowtie$ on $\mathcal{G}_{1}$ with $[c]_{\bowtie}=\{c\}$. A special case is when $\mathcal{G}_{1}$ is an regular network, i.e., a network where every cell is input equivalent with each other. As an example, consider the networks $\mathcal{G}_{1}, \mathcal{G}_{2}$ and $\mathcal{G}_{3}$ in Figure 1 , where $\mathcal{G}_{2}$, which is regular, is obtained from $\mathcal{G}_{1}$ by removing the edge $1 \rightarrow 3$, and $\mathcal{G}_{3}$ is obtained from $\mathcal{G}_{2}$ by removing the edge $4 \rightarrow 1$. Thus, both lattices of balanced relations on $\mathcal{G}_{1}$ and $\mathcal{G}_{3}$ are subsets of the lattice of balanced relations on $\mathcal{G}_{2}$. More precisely, every balanced equivalence relation on $\mathcal{G}_{1}$ is also balanced on $\mathcal{G}_{2}$ and balanced equivalence relations on $\mathcal{G}_{3}$ are precisely the balanced equivalence relations $\bowtie$ on $\mathcal{G}_{2}$ such that cell 1 is not $\bowtie$-equivalent to any other cell.

The rewiring case is addressed in Section 3.2. Here, by a rewiring of a network, we mean a graph operation on edges of the network under which the input equivalence relation is preserved. The main result is given by Theorem 3.6. It should be mentioned that some partial results on rewiring have been obtained by Field in [8], where he considered invariants of a network under repatching and explored the patch equivalence for balanced equivalence relations. More precisely, given a network $\mathcal{G}_{1}$ and an equivalence relation $\bowtie$ on its cell set $C$, a network $\mathcal{G}_{2}$ is called a repatching of $\mathcal{G}_{1}$ if the number of edges, per edge-type, from cells in the $\bowtie$-class of $c$ to every cell in the $\bowtie$-class of cell $d$ is the same on both $\mathcal{G}_{1}$ and $\mathcal{G}_{2}$, for all $c, d \in C$. The networks $\mathcal{G}_{1}$ and $\mathcal{G}_{2}$ are called patch $\bowtie$-equivalent according to the terminology in Field [8]. It follows that for patch $\bowtie$-equivalent networks $\mathcal{G}_{1}$ and $\mathcal{G}_{2}$, the relation $\bowtie$ is balanced on $\mathcal{G}_{2}$ if and only if is balanced on $\mathcal{G}_{1}$ (cf. Field [8]). In Section 3.2, we characterize those balanced equivalence relations on $\mathcal{G}_{1}$ that are also balanced on $\mathcal{G}_{2}$. Moreover, we extend the above result of Field [8] in the following way. Suppose the rewiring operation replaces 
an input edge $d \rightarrow c$ by $a \rightarrow c$, then a balanced equivalence relation $\bowtie$ is simultaneously balanced on $\mathcal{G}_{1}$ and $\mathcal{G}_{2}$ if and only if either of the two occurs: $d$ is $\bowtie$-equivalent to $a$ or $c$ is not $\bowtie$-equivalent with any other cell. (See Corollary 3.8.)

In Section 4 we investigate the effect of operations on nodes. As for the case of edge deletion or addition, there are balanced relations that are inherited from the initial lattice (cf. Theorems 4.4, 4.5 and Corollary 4.6). When a network $\mathcal{G}_{2}$ is obtained from a network $\mathcal{G}_{1}$ by removing a node, together with all its edges, we show that the new lattice of balanced equivalence relations on $\mathcal{G}_{2}$ can be completely inherited from the initial lattice of balanced relations on $\mathcal{G}_{1}$, if the input equivalence relation on $\mathcal{G}_{2}$ refines the (projected) input equivalence relation on $\mathcal{G}_{1}$; that is, if every two input equivalent cells of $\mathcal{G}_{2}$ are also input equivalent for the network $\mathcal{G}_{1}$. In this case, if $c$ denotes the removed node, then the lattice of balanced relations for $\mathcal{G}_{2}$ is obtained from the lattice for $\mathcal{G}_{1}$ by considering the projection of the balanced relations $\bowtie$ on $\mathcal{G}_{1}$ such that $[c]_{\bowtie}=\{c\}$ - the projection is by discarding the singleton $\bowtie$-class of $c$. As special cases, when there are no outgoing edges from $c$ to other cells, or when $\mathcal{G}_{1}$ is a regular network, then the lattice of balanced relations for $\mathcal{G}_{2}$ is completely inherited from the lattice of balanced relations for $\mathcal{G}_{1}$ (cf. Corollary 4.6).

In a near future, based on our results here, we intend to develop an algorithm that given the lattice of balanced equivalence relations of a network generates the lattice of balanced equivalence relations of its evolution by elementary graph operations. We intend to implement that algorithm in the form of a computer program and make it available through a free-access web page, so that it can be executed, for any coupled cell network, by users of general interest.

\section{Preliminaries}

In this section we introduce notations and give a brief review on coupled cell networks, coupled cell systems, synchrony subspaces and balanced equivalence relations on networks. We follow the framework of Stewart, Golubitsky et al. [15, 10], where more details can be found. See also Golubitsky and Stewart [9] for a survey on the subject.

\section{Coupled cell networks and coupled cell systems}

A coupled cell network is a directed graph whose nodes represent the cells and edges describe couplings among the cells. Equivalence relations on nodes and edges are used to indicate the cell types and the edge types and are represented by different shapes of nodes and edges in the graph. A coupled cell network may have multiple edges and loops. Given two network cells $c, d$ and a network edge $e=d \rightarrow c$, the cell $d$ is called the tail cell and $c$ is called the head cell of $e$. The edge $e$ is called an input edge of $c$. The set of all tail cells of input edges of $c$, which is a multiset, is called the input set of $c$, usually denoted by $I(c)$. It is assumed the consistency condition that two equivalent edges have equivalent head cells and equivalent tail cells.

By a multiset, we mean a generalized notion of set, in which elements are allowed to appear more than once. For a multiset $A$ and $x \in A$, define the multiplicity of $x$ as the 
number of copies of $x$ contained in $A$, denoted by $\mathfrak{m}(x, A)$; for a subset $B \subset A$, define the multiplicity of $B$ as $\mathfrak{m}(B, A):=\sum_{x \in B} \mathfrak{m}(x, A)$.

In this work we consider networks with only one cell-equivalence class and one edge-equivalence class. In this case, two network cells are called input equivalent, if the number of input edges is the same for the two cells. Thus two cells $c_{1}, c_{2}$ are input equivalent, denoted by $c_{1} \sim_{I} c_{2}$, if $\# I\left(c_{1}\right)=\# I\left(c_{2}\right)$, where $\# I\left(c_{i}\right)$ denotes the cardinality of the multiset $I\left(c_{i}\right), i=1,2$. It follows from the consistency condition that the input equivalence relation $\sim_{I}$ refines the cell equivalence relation.

A coupled cell network is called regular, if it has only one input-equivalence class and only one edge-equivalence class.

Given a coupled cell network $\mathcal{G}$, associate to every cell $c$ a finite-dimensional real vector space $P_{c}$, called the cell phase space. A coupled cell system is a system of ordinary differential equations whose structure is consistent with the network structure of $\mathcal{G}$ and whose total phase space $P$ is defined by the direct product of all the cell phase spaces.

\section{Synchrony subspaces and balanced equivalence relations}

The concept of balanced equivalence relations on coupled cell networks is closely related to synchrony subspaces admitted by admissible coupled cell systems. The following definition follows [10]. Given a network $\mathcal{G}$ with only one edge-type, an equivalence relation $\bowtie$ defined on its set of cells is called balanced on $\mathcal{G}$ if for every two cells $c, d$ with $c \bowtie d$, there exists an isomorphism $\beta$ between their input sets $I(c)$ and $I(d)$ preserving the $\bowtie$ equivalence relations: for all $i \in I(c)$ we have $i \bowtie \beta(i)$. It follows from the definition that any balanced equivalence relation refines the input equivalence relation $\sim_{I}$.

Using the multisets and multiplicity, we have the following equivalent definition of a balanced equivalence relation on a network.

Definition 2.1 Given a network $\mathcal{G}$, an equivalence relation $\bowtie$ defined on the network set of cells is called balanced, if for every two cells $c, d$ with $c \bowtie d$, we have

$$
\mathfrak{m}\left([\alpha]_{\bowtie}, I(c)\right)=\mathfrak{m}\left([\alpha]_{\bowtie}, I(d)\right), \quad \text { for all cell } \alpha \text {. }
$$

Given a network, an equivalence relation $\bowtie$ on its set of cells and a choice of the total phase space $P$ for the network, the polydiagonal subspace associated with $\bowtie$, denoted by $\Delta_{\bowtie}$, is the subspace of $P$ defined in terms of the cell coordinates in the following way: if two cells are $\bowtie$ related then it is assumed equality of the corresponding cell coordinates. Moreover, the polydiagonal subspace $\Delta_{\triangleright}$ of $P$ is called a synchrony subspace if it is flow-invariant for all $\mathcal{G}$-admissible vector fields on $P$.

In [10] it is proved that, given a coupled cell network, an equivalence relation $\bowtie$ on its set of cells and a choice $P$ of the total phase space, the polydiagonal subspace $\Delta_{\bowtie}$ is a synchrony subspace of the network if and only if $\bowtie$ is balanced.

Given a network $\mathcal{G}$ with the set $C$ of cells, we denote by $M_{\mathcal{G}}$ the set of all equivalence

relations on $C$ and by $\Lambda_{\mathcal{G}}$ the set of all balanced equivalence relations on $C$. Both sets 
have a partially ordered structure, using the relation of refinement $<$ defined as: for two equivalence relations $\bowtie_{i}$ and $\bowtie_{j}$, we say $\bowtie_{i}$ refines $\bowtie_{j}$, written as $\bowtie_{i} \prec \bowtie_{j}$, if

$$
[c]_{i} \subseteq[c]_{j} \quad \forall c \in C .
$$

Here, $[c]_{l}$ denotes the $\bowtie_{l}$-equivalence class of cell $c$, for $l=i, j$.

As pointed out in [14], both sets of balanced equivalence relations and synchrony subspaces for a given network are complete lattices, with respect to the relation of refinement and the inclusion of subspaces, respectively. See [2, Section 3] for a compact discussion on this topic.

\section{Graph Operations on Edges}

In this section, we consider three kinds of operations on the edges of a network: deletion, addition and rewiring. Our aim is to describe the common balanced equivalence relations of two networks when they are related by these operations.

We start by addressing the deletion and addition of edges and then the rewiring case. We note that, although the rewiring of an edge can be considered as a consecutive composition of removing one edge from the input set of a cell and then adding another edge to that set, it is more advantageous to consider the rewiring as a one-step operation since, this way, we can get stronger results.

\subsection{Deleting and Adding Edges}

Let $\mathcal{G}_{2}$ be a network obtained from a network $\mathcal{G}_{1}$ by:

$$
\begin{array}{cl}
\text { Removing } s \text { edges: } & d_{1} \rightarrow c_{1}, \cdots, d_{s} \rightarrow c_{s} ; \\
\text { Adding } r \text { edges: } & b_{1} \rightarrow a_{1}, \cdots b_{r} \rightarrow a_{r} .
\end{array}
$$

Here, $r, s$ are nonnegative integers. Also, we are assuming that $c_{i} \neq a_{j}$ for all $i, j$, that is, there is no rewiring occurring in (3.2)-(3.3).

Denote by $\mathcal{G}$ the network obtained from $\mathcal{G}_{1}$ by removing the edges in (3.2) and consider the networks

$$
\mathcal{G}_{E}:=\mathcal{G}_{1}-\mathcal{G}_{,} \quad \mathcal{G}_{F}:=\mathcal{G}_{2}-\mathcal{G}
$$

Theorem 3.1 Let $\mathcal{G}_{2}$ be obtained from $\mathcal{G}_{1}$ by (3.2)-(3.3). A relation is balanced on both $\mathcal{G}_{1}$ and $\mathcal{G}_{2}$ if and only if it is balanced on both $\mathcal{G}_{E}$ and $\mathcal{G}_{F}$.

Proof Assume (3.2)-(3.3) and take for example a balanced relation $\bowtie$ on $\mathcal{G}_{1}$. We prove that it is also balanced on $\mathcal{G}_{2}$ if and only if it is balanced on both $\mathcal{G}_{E}$ and $\mathcal{G}_{F}$. Given that

$$
I_{1}(x)=I_{2}(x)+I_{E}(x)-I_{F}(x), \quad \forall x \in C,
$$

we have

$$
\mathfrak{m}\left([\alpha]_{\bowtie}, I_{1}(x)\right)=\mathfrak{m}\left([\alpha]_{\bowtie}, I_{2}(x)\right)+\mathfrak{m}\left([\alpha]_{\bowtie}, I_{E}(x)\right)-\mathfrak{m}\left([\alpha]_{\bowtie}, I_{F}(x)\right), \quad \forall \alpha \in C .
$$


Since $\bowtie$ is balanced, for $x \bowtie y$, we have

$$
\mathfrak{m}\left([\alpha]_{\bowtie}, I_{1}(x)\right)=\mathfrak{m}\left([\alpha]_{\bowtie}, I_{1}(y)\right), \quad \forall \alpha \in C .
$$

By assumption, there is no rewiring, so we have

$$
\mathfrak{m}\left([\alpha]_{\bowtie}, I_{E}(x)\right)=0 \quad \text { or } \quad \mathfrak{m}\left([\alpha]_{\bowtie}, I_{F}(x)\right)=0, \quad \forall \alpha, x \in C .
$$

Therefore, we can conclude from (3.4), that

$$
\mathfrak{m}\left([\alpha]_{\bowtie}, I_{2}(x)\right)=\mathfrak{m}\left([\alpha]_{\bowtie}, I_{2}(y)\right)
$$

if and only if

$$
\mathfrak{m}\left([\alpha]_{\bowtie}, I_{E}(x)\right)=\mathfrak{m}\left([\alpha]_{\bowtie}, I_{E}(y)\right) \quad \text { and } \quad \mathfrak{m}\left([\alpha]_{\bowtie}, I_{F}(x)\right)=\mathfrak{m}\left([\alpha]_{\bowtie}, I_{F}(y)\right) .
$$

That is, $\bowtie$ is balanced on $\mathcal{G}_{2}$ if and only if it is balanced on both $\mathcal{G}_{E}$ and $\mathcal{G}_{F}$.

Using the fact that every balanced equivalence relation must be a refinement of the input equivalence relation, we get stronger results for the case of simply deleting (or adding) a single edge. Note that the input equivalence itself is easy to identify from the graph. We denote by $\sim_{I_{i}}$ the input equivalence relation on $\mathcal{G}_{i}$, for $i=1,2$.

Corollary 3.2 Let $\mathcal{G}_{2}$ be obtained from $\mathcal{G}_{1}$ by deleting (or adding) a single edge $\rightarrow c$. Then every balanced equivalence relation $\bowtie$ on $\mathcal{G}_{1}$ such that $[c]_{\bowtie}=\{c\}$ is balanced on $\mathcal{G}_{2}$. Moreover, if $[c]_{\sim_{I_{2}}}=\{c\}$ then these are the only balanced relations on $\mathcal{G}_{2}$.

Proof We give the proof for the case of deleting the single edge $d \rightarrow c$, as the proof for the adding case is analogous. In this case, $\mathcal{G}_{F}$ is a network of isolated cells and $\mathcal{G}_{E}$ is a network of isolated cells except for $c$ which has an input edge from $d$. Trivially, every equivalence relation on $\mathcal{G}_{F}$ is balanced and the balanced equivalence relations on $\mathcal{G}_{E}$ are precisely those where $c$ is only equivalent with itself, since $c$ forms a single input equivalent class on its own. In summary, we have in this case,

$\bowtie$ is balanced on both $\mathcal{G}_{E}$ and $\mathcal{G}_{F} \Leftrightarrow \bowtie$ is balanced on $\mathcal{G}_{E} \quad \Leftrightarrow \quad[c]_{\bowtie}=\{c\}$.

Let $\bowtie$ be balanced on $\mathcal{G}_{1}$ and such that $[c]_{\bowtie}=\{c\}$. Then, by (3.5), we have $\bowtie$ is balanced on $\mathcal{G}_{E}$ and on $\mathcal{G}_{F}$. It follows from Theorem 3.1 that $\bowtie$ is balanced on $\mathcal{G}_{2}$.

If $[c]_{\sim_{I_{2}}}=\{c\}$ and $\bowtie$ is balanced on $\mathcal{G}_{2}$, then $[c]_{\bowtie}=\{c\}$, since $\bowtie$ must refine $\sim_{I_{2}}$. Thus, by (3.5), we have that $\bowtie$ is balanced of both $\mathcal{G}_{E}$ and $\mathcal{G}_{F}$. It follows from Theorem 3.1 that $\bowtie$ is balanced on $\mathcal{G}_{1}$.

We discuss a special case of Corollary 3.2. Observing that

$$
[c]_{\sim_{I_{1}}} \cap[c]_{\sim_{I_{2}}}=\{c\},
$$

if $\sim_{I_{2}}$ refines $\sim_{I_{1}}$, then $[c]_{\sim_{I_{2}}}=\{c\}$. Thus we have the following:

Corollary 3.3 Let $\mathcal{G}_{2}$ be obtained from $\mathcal{G}_{1}$ by deleting (or adding) a single edge $d \rightarrow c$. If the input relation on $\mathcal{G}_{2}$ refines the input relation on $\mathcal{G}_{1}$, then the balanced relations on $\mathcal{G}_{2}$ are the balanced relations $\bowtie$ on $\mathcal{G}_{1}$ such that $[c]_{\bowtie}=\{c\}$. 
Corollary 3.3 is especially effective when $\mathcal{G}_{1}$ is a regular network, and so all cells are input isomorphic. See Example 3.4.

Example 3.4 Consider a network whose topology changes over time, as shown in Figure 1 . The network $\mathcal{G}_{2}$ is obtained from $\mathcal{G}_{1}$ by removing the edge $1 \rightarrow 3$, and $\mathcal{G}_{3}$ is obtained from $\mathcal{G}_{2}$ by removing the edge $4 \rightarrow 1$. The sets of the balanced equivalence relations on $\mathcal{G}_{i}$ are listed in Table 1.

In the first step, the edge $1 \rightarrow 3$ is removed from $\mathcal{G}_{1}$. Note that every balanced equivalence relation $\bowtie$ on $\mathcal{G}_{1}$ satisfies [3] $]_{\bowtie}=\{3\}$. Thus, by Corollary 3.2, every balanced equivalence relation $\bowtie$ on $\mathcal{G}_{1}$ is also balanced on $\mathcal{G}_{2}$. That is, the relations $\bowtie_{0}^{1}, \bowtie_{1}^{1}, \bowtie_{2}^{1}$ in Table 1 are balanced on $\mathcal{G}_{2}$. In the next step, the edge $4 \rightarrow 1$ is removed from $\mathcal{G}_{2}$. Since $\mathcal{G}_{2}$ is a regular network, we have that $\sim_{I_{3}}$ refines $\sim_{I_{2}}$. Thus, by Corollary 3.3, every balanced relation on $\mathcal{G}_{3}$ is a balanced relation on $\mathcal{G}_{2}$ satisfying [1] $]_{\bowtie}=\{1\}$. That is, the only balanced relations on $\mathcal{G}_{3}$ are $\bowtie_{0}^{2}$ and $\bowtie_{5}^{2}$ of Table 1 .

\begin{tabular}{|c|c|c|}
\hline$\Lambda_{\mathcal{G}_{1}}$ & $\Lambda_{\mathcal{G}_{2}}$ & $\Lambda_{\mathcal{G}_{3}}$ \\
\hline $\begin{array}{l}\bowtie_{0}^{1}=\{\{1\},\{2\},\{3\},\{4\},\{5\}\} \\
\bowtie_{1}^{1}=\{\{1,2\},\{3\},\{4,5\}\} \\
\bowtie_{2}^{1}=\{\{1\},\{2\},\{3\},\{4,5\}\}\end{array}$ & $\begin{array}{l}\bowtie_{0}^{2}=\{\{1\},\{2\},\{3\},\{4\},\{5\}\}=\bowtie_{0}^{1} \\
\bowtie_{1}^{2}=\{\{1,2\},\{3\},\{4,5\}\}=\triangleleft_{1}^{1} \\
\bowtie_{2}^{2}=\{\{1,3\},\{2,5\},\{4\}\} \\
\bowtie_{3}^{2}=\{\{1,3,4\},\{2,5\}\} \\
\bowtie_{4}^{2}=\{\{1,3\},\{2,4,5\}\} \\
\bowtie_{5}^{2}=\{\{1\},\{2\},\{3\},\{4,5\}\}=\bowtie_{2}^{1} \\
\bowtie_{6}^{2}=\{\{1,2,3,4,5\}\}\end{array}$ & $\begin{array}{l}\bowtie_{0}^{3}=\{\{1\},\{2\},\{3\},\{4\},\{5\}\}=\bowtie_{0}^{1} \\
\bowtie_{1}^{3}=\{\{1\},\{2\},\{3\},\{4,5\}\}=\bowtie_{2}^{1}\end{array}$ \\
\hline
\end{tabular}

Table 1: The balanced equivalence relations for the networks $\mathcal{G}_{1}, \mathcal{G}_{2}, \mathcal{G}_{3}$ in Figure 1.

Remark 3.5 It should be mentioned that the case where an edge changes its edge type (for networks with more than one edge type) can be similarly treated, since it can be interpreted as a composition of first deleting an edge of one type and then adding an edge of another type. Note that this changes the relation of input equivalence among the cells. One shows similarly that every equivalence relation for which the head cell of the changing edge forms a single class remains balanced.

\subsection{Rewiring of Edges}

Let $\mathcal{G}_{2}$ be obtained from $\mathcal{G}_{1}$ by a rewiring corresponding, for $i=1,2, \ldots, n$, to:

Removing $m_{i}$ edges directed to cell $c_{i}: d_{1}^{i} \rightarrow c_{i}, \cdots, d_{m_{i}}^{i} \rightarrow c_{i}$;

Adding $m_{i}$ edges directed to cell $c_{i}: a_{1}^{i} \rightarrow c_{i}, \cdots, a_{m_{i}}^{i} \rightarrow c_{i}$.

Put

$$
D_{i}:=\left\{d_{1}^{i}, \ldots, d_{m_{i}}^{i}\right\}, \quad A_{i}:=\left\{a_{1}^{i}, \ldots, a_{m_{i}}^{i}\right\}
$$


and note that we are assuming the cardinality of $D_{i}$ and $A_{i}$ equal as multiset (so that they pair up as rewirings).

Theorem 3.6 Let $\mathcal{G}_{1}$ and $\mathcal{G}_{2}$ be two networks with set of cells $C$ and where $\mathcal{G}_{2}$ is obtained from $\mathcal{G}_{1}$ by a rewiring corresponding, for $i=1,2, \ldots, n$, to (3.7)-(3.8). Take $D_{i}$ and $A_{i}$ as above, where $\# D_{i}=\# A_{i}$ as multisets. Every balanced equivalence relation $\bowtie$ on $\mathcal{G}_{1}$ that satisfies, for $i=1, \ldots, n$, one of the conditions

(i) $\left[c_{i}\right]_{\bowtie}=\left\{c_{i}\right\}$, or

(ii) $\#\left(D_{i} \cap[\alpha]_{\bowtie}\right)=\#\left(A_{i} \cap[\alpha]_{\bowtie}\right), \quad \forall \alpha \in C$, or

(iii) $\left[c_{i}\right]_{\bowtie} \subset\left\{c_{1}, \cdots, c_{n}\right\}$ and $\#\left(A_{i} \cap[\alpha]_{\bowtie}\right)-\#\left(D_{i} \cap[\alpha]_{\bowtie}\right)=\#\left(A_{j} \cap[\alpha]_{\bowtie}\right)-\#\left(D_{j} \cap[\alpha]_{\bowtie}\right), \quad \forall \alpha \in C, \forall c_{j} \in\left[c_{i}\right]_{\bowtie,}$

is also balanced on $\mathcal{G}_{2}$.

Proof Let $\bowtie$ be a balanced equivalence relation on $\mathcal{G}_{1}$. We prove that if $\bowtie$ satisfies one of the conditions (i)-(iii) then it is also balanced on $\mathcal{G}_{2}$. For this, we have to show that, for all $x, y$ such that $x \bowtie y$,

$$
m\left([\alpha]_{\bowtie}, I_{2}(x)\right)=m\left([\alpha]_{\bowtie}, I_{2}(y)\right), \forall \alpha \in C
$$

knowing that this is valid for $I_{1}(x)$ and $I_{1}(y)$, respectively.

Since, for the cells $c \in C \backslash\left\{c_{1}, \ldots, c_{n}\right\}$, we have $I_{2}(c)=I_{1}(c)$, in what follows we just need to consider the cells $c_{i}, i=1, \ldots, n$.

If condition (i) holds then we have nothing to check. If the equality given by condition (ii) holds then, since $I_{2}\left(c_{i}\right)=I_{1}\left(c_{i}\right) \backslash D_{i} \cup A_{i}$, we have

$$
m\left([\alpha]_{\bowtie}, I_{2}\left(c_{i}\right)\right)=m\left([\alpha]_{\bowtie}, I_{1}\left(c_{i}\right)\right), \forall \alpha \in C
$$

and thus (3.9) follows trivially for every $x, y \in\left[c_{i}\right]_{\bowtie}$.

If condition (iii) holds then, given that $I_{2}\left(c_{i}\right)=I_{1}\left(c_{i}\right) \backslash D_{i} \cup A_{i}$, the equality

$$
\#\left(A_{i} \cap[\alpha]_{\bowtie}\right)-\#\left(D_{i} \cap[\alpha]_{\bowtie}\right)=\#\left(A_{j} \cap[\alpha]_{\bowtie}\right)-\#\left(D_{j} \cap[\alpha]_{\bowtie}\right) \quad \forall \alpha \in C
$$

together with

$$
m\left([\alpha]_{\bowtie}, I_{1}\left(c_{i}\right)\right)=m\left([\alpha]_{\bowtie}, I_{2}\left(c_{j}\right)\right), \forall \alpha \in C
$$

implies the equality (3.9) for every $x, y \in\left[c_{i}\right]_{\bowtie \triangleleft}$.

In case $\mathcal{G}_{2}$ is obtained from $\mathcal{G}_{1}$ by a rewiring corresponding to deleting and adding an equal number of edges from the input set of a unique cell $c$, the statement of Theorem 3.6 is:

Corollary 3.7 Let $\mathcal{G}_{1}$ and $\mathcal{G}_{2}$ be two networks with set of cells $C$ and where $\mathcal{G}_{2}$ is obtained from $\mathcal{G}_{1}$ by a rewiring corresponding to removing $m$ edges directed to cell $c$ and adding $m$ edges directed to cell c. As before, take $D$ and $A$ the set of the head cells involved in the removing and adding edges, respectively. Every balanced equivalence relation $\bowtie$ on $\mathcal{G}_{1}$ that satisfies one of the conditions 
(i) $[c]_{\bowtie}=\{c\}$, or

(ii) $\#\left(D \cap[\alpha]_{\bowtie}\right)=\#\left(A \cap[\alpha]_{\bowtie}\right), \quad \forall \alpha \in C$,

is also balanced on $\mathcal{G}_{2}$.

Corollary 3.8 Let $\mathcal{G}_{1}$ and $\mathcal{G}_{2}$ be two networks with set of cells $C$ and where $\mathcal{G}_{2}$ is obtained from $\mathcal{G}_{1}$ by a rewiring corresponding to deleting from the input set of a unique cell $c$ the edge $d \rightarrow c$ and adding the edge $a \rightarrow c$. Every balanced equivalence relation $\bowtie$ on $\mathcal{G}_{1}$ that satisfies one of the conditions, $[c]_{\bowtie}=\{c\}$ or $d \bowtie a$, is also balanced on $\mathcal{G}_{2}$.

Remark 3.9 Corollary 3.8 is an extension of Remark 14 (2) in Field [8].

Example 3.10 Consider the networks $\mathcal{G}_{1}$ and $\mathcal{G}_{2}$ given in Figure 2, where $\mathcal{G}_{2}$ is obtained from $\mathcal{G}_{1}$ by a rewiring corresponding to deleting from the input set of cell 2 the edge $3 \rightarrow 2$ and adding the edge $4 \rightarrow 2$. Note that both $\mathcal{G}_{1}, \mathcal{G}_{2}$ are regular. Thus, all cells are input equivalent. By Corollary 3.8, balanced equivalence relations on $\mathcal{G}_{1}$ that either satisfy $[2]_{\bowtie}=\{2\}$ or $3 \bowtie 4$ are balanced again on $\mathcal{G}_{2}$. The balanced equivalence relations on $\mathcal{G}_{1}$ are presented in Table 2. Thus those satisfying [2] $]_{\bowtie}=\{2\}$ are $\bowtie_{0}^{1}, \bowtie_{1}^{1}, \bowtie_{3}^{1}, \bowtie_{6}^{1}$; and those such that $3 \bowtie 4$ are $\bowtie_{7}^{1}, \bowtie_{9}^{1}, \bowtie_{10}^{1}, \bowtie_{11}^{1}, \bowtie_{12}^{1}$. The balanced equivalence relations on $\mathcal{G}_{2}$ are presented in Table 3.
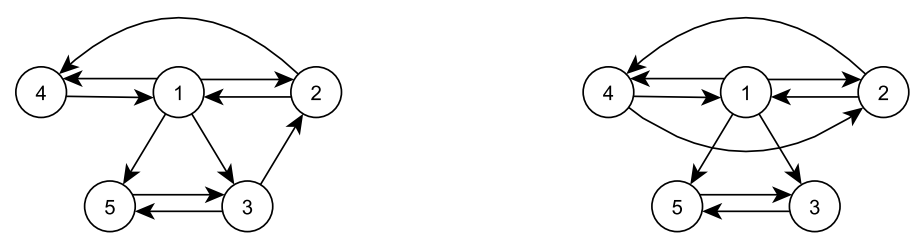

$\mathcal{G}_{1}$

Figure 2: An example of rewiring the edge $3 \rightarrow 2$ to $4 \rightarrow 2$ on $\mathcal{G}_{1}$ to obtain $\mathcal{G}_{2}$.

\begin{tabular}{|c|c|c|}
\hline $\begin{array}{l}\bowtie_{0}^{1}=\{\{1\},\{2\},\{3\},\{4\},\{5\}\} \\
\bowtie_{1}^{1}=\{\{1,4\},\{2\},\{3\},\{5\}\} \\
\bowtie_{2}^{1}=\{\{1\},\{2,5\},\{3\},\{4\}\} \\
\bowtie_{3}^{1}=\{\{1\},\{2\},\{3,5\},\{4\}\}\end{array}$ & $\begin{array}{l}\bowtie_{4}^{1}=\{\{1\},\{2,3,5\},\{4\}\} \\
\bowtie_{5}^{1}=\{\{1,4\},\{2,5\},\{3\}\} \\
\bowtie_{6}^{1}=\{\{1,4\},\{3,5\},\{2\}\} \\
\bowtie_{7}^{1}=\{\{1\},\{2,5\},\{3,4\}\}\end{array}$ & $\begin{array}{l}\bowtie_{8}^{1}=\{\{1,4\},\{2,3,5\}\} \\
\triangleleft_{9}^{1}=\{\{1,2,5\},\{3,4\}\} \\
\triangleleft_{10}^{1}=\{\{1,3,4\},\{2,5\}\} \\
\triangleleft_{11}^{1}=\{\{1\},\{2,3,4,5\}\} \\
\triangleleft_{12}^{1}=\{\{1,2,3,4,5\}\}\end{array}$ \\
\hline
\end{tabular}

Table 2: The balanced equivalence relations for the network $\mathcal{G}_{1}$ of Figure 2.

\section{Graph Operations on Nodes}

In this section, we analyze the influence of the graph operations deletion and addition of nodes on the lattice of balanced equivalence relations of a network. We assume that 


\begin{tabular}{|c|c|c|}
\hline $\begin{array}{l}\bowtie_{0}^{2}=\{\{1\},\{2\},\{3\},\{4\},\{5\}\}=\bowtie_{0}^{1} \\
\bowtie_{1}^{2}=\{\{1,2\},\{3\},\{4\},\{5\}\} \\
\bowtie_{2}^{2}=\{\{1,4\},\{2\},\{3\},\{5\}\}=\bowtie_{1}^{1} \\
\bowtie_{3}^{2}=\{\{1\},\{2,4\},\{3\},\{5\}\} \\
\bowtie_{4}^{2}=\{\{1\},\{2\},\{3,5\},\{4\}\}=\bowtie_{3}^{1}\end{array}$ & $\begin{array}{l}\bowtie_{5}^{2}=\{\{1,2,4\},\{3\},\{5\}\} \\
\bowtie_{6}^{2}=\{\{1\},\{2,3\},\{4,5\}\} \\
\bowtie_{7}^{2}=\{\{1,2\},\{3,5\},\{4\}\} \\
\bowtie_{8}^{2}=\{\{1\},\{2,4\},\{3,5\}\} \\
\triangleleft_{9}^{2}=\{\{1\},\{2,5\},\{3,4\}\}=\triangleleft_{7}^{1} \\
{\triangleleft_{10}^{2}}_{10}=\{\{1,4\},\{2\},\{3,5\}\}=\triangleleft_{6}^{1}\end{array}$ & 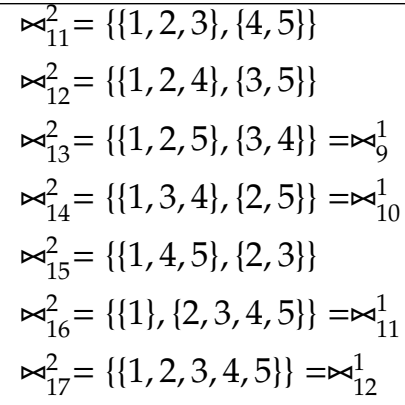 \\
\hline
\end{tabular}

Table 3: The balanced equivalence relations for the network $\mathcal{G}_{2}$ of Figure 2.

we have two networks $\mathcal{G}_{1}, \mathcal{G}_{2}$ that are related by an addition/ deletion of a node. Say, $\mathcal{G}_{2}$ is related with $\mathcal{G}_{1}$ by removing a node $c$ together with its incoming and outgoing edges. Naturally, in order to relate balanced equivalence relations on $\mathcal{G}_{1}$ and on $\mathcal{G}_{2}$, we have to define projection and lifting of partitions at the corresponding sets of cells. More precisely, we define:

Definition 4.1 Let $C_{1}$ be a set of cells and $C_{2}=C_{1} \backslash\{c\}$ for some $c \in C_{1}$. Let $\mathrm{M}_{i}$ be the set of all equivalence relations on $C_{i}$ for $i=1,2$.

(i) The map of projection is defined by Proj: $\mathrm{M}_{1} \rightarrow \mathrm{M}_{2}$ where, if $\bowtie \in \mathrm{M}_{1}$ :

$$
[\alpha]_{\operatorname{Proj}(\bowtie)}= \begin{cases}{[\alpha]_{\bowtie} \backslash\{c\}} & \text { if } c \in[\alpha]_{\bowtie} \\ {[\alpha]_{\bowtie}} & \text { otherwise. }\end{cases}
$$

(ii) The map of lifting is defined by Lift : $\mathrm{M}_{2} \rightarrow \mathrm{M}_{1}$ where if $\bowtie \in \mathrm{M}_{2}$ :

$$
[\alpha]_{\mathrm{Lift}(\bowtie)}= \begin{cases}\{c\} & \text { if } \alpha=c \\ {[\alpha]_{\bowtie}} & \text { otherwise. }\end{cases}
$$

\subsection{Deletion/Addition of a Node}

Let $\mathcal{G}_{1}, \mathcal{G}_{2}$ be two coupled cell networks where $\mathcal{G}_{2}$ is the network obtained from $\mathcal{G}_{1}$ by removing a node $c$ from $\mathcal{G}_{1}$ together with its incoming and outgoing edges of $c$. Equivalently, $\mathcal{G}_{1}$ is obtained from $\mathcal{G}_{2}$ by adding a node and (some) incoming and outgoing edges. We will use the following terminology:

Definition 4.2 A balanced equivalence relation on $\mathcal{G}_{2}$ is said to be inherited from $\mathcal{G}_{1}$, if it is the projection of a balanced equivalence relation on $\mathcal{G}_{1}$. We denote by $\Lambda_{\mathcal{G}_{2}}^{I}$ the set of all balanced equivalence relations on $\mathcal{G}_{2}$ that are inherited from $\mathcal{G}_{1}$. Similarly, a balanced equivalence relation on $\mathcal{G}_{1}$ is said to be inherited from $\mathcal{G}_{2}$, if it is the lift of a balanced equivalence relation on $\mathcal{G}_{2}$.

We describe now the set of all balanced equivalence relations on $\mathcal{G}_{2}$ (on $\mathcal{G}_{1}$ ) that are inherited from $\mathcal{G}_{1}$ (from $\mathcal{G}_{2}$ ). The first question we may ask is if the projection of 
a balanced equivalence relation on $\mathcal{G}_{1}$ is also a balanced equivalence relation on $\mathcal{G}_{2}$. Similarly, we can ask if the lift of a balanced equivalence relation on $\mathcal{G}_{2}$ is balanced on $\mathcal{G}_{1}$. Trivially, a necessary condition for a balanced equivalence relation on $\mathcal{G}_{2}$ to be inherited from a balanced relation $\bowtie$ on $\mathcal{G}_{1}$ is that the projection of $\bowtie$ refines the input relation of $\mathcal{G}_{2}$, since every balanced relation on a network has to refine the network input relation. Similarly, a necessary condition for the lift of a balanced equivalence relation on $\mathcal{G}_{2}$ to be a balanced relation on $\mathcal{G}_{1}$ is that such lift refines the input relation of $\mathcal{G}_{1}$. In fact, we show next that those conditions are also sufficient. That is:

Lemma 4.3 Let $\mathcal{G}_{2}$ be obtained from $\mathcal{G}_{1}$ by removing a cell $c$.

(i) The projection of a balanced equivalence relation $\bowtie$ on $\mathcal{G}_{1}$ is balanced on $\mathcal{G}_{2}$ if and only if it refines the input relation on $\mathcal{G}_{2}$.

(ii) The lift of a balanced equivalence relation $\bowtie$ on $\mathcal{G}_{2}$ is balanced on $\mathcal{G}_{1}$ if and only if it refines the input relation on $\mathcal{G}_{1}$.

Proof (i) Let $\bowtie$ be a balanced equivalence relation on $\mathcal{G}_{1}$ such that $\operatorname{Proj}(\bowtie) \prec \sim_{I_{2}}$. Need to show only that $\operatorname{Proj}(\bowtie)$ is balanced on $\mathcal{G}_{2}$. For convenience, denote by $\bowtie^{\prime}=\operatorname{Proj}(\bowtie)$. We need to show

$$
\mathfrak{m}\left([\alpha]_{\triangleright^{\prime}}, I_{2}(x)\right)=\mathfrak{m}\left([\alpha]_{\triangleright^{\prime}}, I_{2}(y)\right), \quad \forall \alpha \in C_{2},
$$

whenever $x, y \in C_{2}$ are such that $x \triangleright^{\prime} y$ and $x \neq y$.

Let $x, y \in C_{2}$ be such that $x \triangleleft^{\prime} y$ and $x \neq y$. Then, by definition of projection, we have $x \bowtie y$. As $\bowtie$ is balanced on $\mathcal{G}_{1}$, we have

$$
\mathfrak{m}\left([\alpha]_{\bowtie}, I_{1}(x)\right)=\mathfrak{m}\left([\alpha]_{\bowtie}, I_{1}(y)\right), \quad \forall \alpha \in C_{1} .
$$

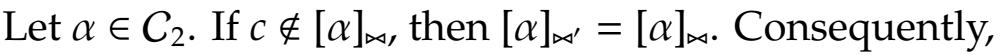

$$
\begin{aligned}
\mathfrak{m}\left([\alpha]_{\triangleright^{\prime}}, I_{2}(x)\right) & =\mathfrak{m}\left([\alpha]_{\bowtie,}, I_{1}(x)-\{c\}\right)=\mathfrak{m}\left([\alpha]_{\bowtie,}, I_{1}(x)\right)=\mathfrak{m}\left([\alpha]_{\bowtie}, I_{1}(y)\right) \\
& =\mathfrak{m}\left([\alpha]_{\bowtie}, I_{1}(y)-\{c\}\right)=\mathfrak{m}\left([\alpha]_{\triangleright^{\prime}}, I_{2}(y)\right) .
\end{aligned}
$$

Otherwise if $c \in[\alpha]_{\bowtie}$, then $[\alpha]_{\triangleleft^{\prime}}=[\alpha]_{\bowtie}-\{c\}$. Since $\bowtie^{\prime}<\sim_{I_{2}}$ and $x \bowtie^{\prime} y$, we have $x \sim_{I_{2}} y$. On the other hand, $x \sim_{I_{1}} y$ since $x \bowtie y$ and $\bowtie$ is balanced on $\mathcal{G}_{1}$. Thus,

$$
\mathfrak{m}\left(\{c\}, I_{1}(x)-I_{2}(x)\right)=\mathfrak{m}\left(\{c\}, I_{1}(y)-I_{2}(y)\right),
$$

but $c \notin I_{2}(x)$ for any $x, y \in C_{2}$, therefore, we have

$$
\mathfrak{m}\left(\{c\}, I_{1}(x)\right)=\mathfrak{m}\left(\{c\}, I_{1}(y)\right) .
$$

Combined with (4.10), this leads to

$$
\mathfrak{m}\left([\alpha]_{\bowtie}-\{c\}, I_{1}(x)\right)=\mathfrak{m}\left([\alpha]_{\bowtie}-\{c\}, I_{1}(y)\right) .
$$

Therefore,

$$
\begin{aligned}
\mathrm{m}\left([\alpha]_{\varpi^{\prime}}, I_{2}(x)\right) & =\mathfrak{m}\left([\alpha]_{\bowtie}-\{c\}, I_{1}(x)-\{c\}\right)=\mathfrak{m}\left([\alpha]_{\bowtie}-\{c\}, I_{1}(x)\right) \\
& =\mathfrak{m}\left([\alpha]_{\bowtie}-\{c\}, I_{1}(y)\right)=\mathfrak{m}\left([\alpha]_{\bowtie}-\{c\}, I_{1}(y)-\{c\}\right)=\mathfrak{m}\left([\alpha]_{\bowtie^{\prime}}, I_{2}(y)\right) .
\end{aligned}
$$


(ii) Let $\bowtie$ be a balanced equivalence relation on $\mathcal{G}_{2}$ such that $\operatorname{Lift}(\bowtie) \prec \sim_{I_{1}}$. Need to show only that $\operatorname{Lift}(\bowtie)$ is balanced on $\mathcal{G}_{1}$. For convenience, denote by $\tilde{\bowtie}=\operatorname{Lift}(\bowtie)$.

Let $x, y \in C_{1}$ be such that $x \tilde{凶} y$ and $x \neq y$. Then, by definition of lifting, $x, y \neq c$. We need to show that

$$
\mathfrak{m}\left([\alpha]_{\tilde{\varpi}}, I_{1}(x)\right)=\mathfrak{m}\left([\alpha]_{\tilde{\omega}}, I_{1}(y)\right), \quad \forall \alpha \in C_{1} .
$$

Note that, since $x \bowtie y$ and $\bowtie$ is balanced, we have

$$
\mathfrak{m}\left([\alpha]_{\bowtie,} I_{2}(x)\right)=\mathfrak{m}\left([\alpha]_{\bowtie,} I_{2}(y)\right), \quad \forall \alpha \in C_{2} .
$$

Let $\alpha \in C_{1}$. If $\alpha \neq c$, then $[\alpha]_{\tilde{\omega}}=[\alpha]_{\bowtie \text {. Thus, }}$.

$$
\begin{aligned}
\mathfrak{m}\left([\alpha]_{\check{\varpi},} I_{1}(x)\right) & =\mathfrak{m}\left([\alpha]_{\triangleright \triangleleft}, I_{1}(x)-\{c\}\right)=\mathfrak{m}\left([\alpha]_{\bowtie}, I_{2}(x)\right)=\mathfrak{m}\left([\alpha]_{\bowtie}, I_{2}(y)\right) \\
& =\mathfrak{m}\left([\alpha]_{\bowtie}, I_{1}(y)-\{c\}\right)=\mathfrak{m}\left([\alpha]_{\varpi}, I_{1}(y)\right) .
\end{aligned}
$$

Otherwise if $\alpha=c$, then $[\alpha]_{\tilde{\ltimes}}=\{c\}$. Thus,

$$
\mathfrak{m}\left([c]_{\tilde{凶}}, I_{1}(x)\right)=\mathfrak{m}\left(\{c\}, I_{1}(x)\right)=\mathfrak{m}\left(\{c\}, I_{1}(x)-I_{2}(x)\right)
$$

and

$$
\mathfrak{m}\left([c]_{\tilde{山}}, I_{1}(y)\right)=\mathfrak{m}\left(\{c\}, I_{1}(y)\right)=\mathfrak{m}\left(\{c\}, I_{1}(y)-I_{2}(y)\right) .
$$

Since $\tilde{\bowtie} \prec \sim_{I_{1}}$, it follows that $x \sim_{I_{1}} y$ and since $\bowtie<\sim_{I_{2}}$, we have $x \sim_{I_{2}} y$. Thus,

$$
\mathfrak{m}\left(\{c\}, I_{1}(x)-I_{2}(x)\right)=\mathfrak{m}\left(\{c\}, I_{1}(y)-I_{2}(y)\right)
$$

and so

$$
\mathfrak{m}\left([c]_{\tilde{凶},}, I_{1}(x)\right)=\mathfrak{m}\left([c]_{\tilde{凶}}, I_{1}(y)\right) .
$$

Using Lemma 4.3, we give a description of the set of all balanced equivalence relations on $\mathcal{G}_{2}$ (on $\mathcal{G}_{1}$ ) that are inherited from $\mathcal{G}_{1}$ (from $\mathcal{G}_{2}$ ):

Theorem 4.4 Let $\mathcal{G}_{2}$ be a network obtained from $\mathcal{G}_{1}$ by removing a cell $c$.

(i) The set of balanced equivalence relations on $\mathcal{G}_{2}$ that are inherited from $\mathcal{G}_{1}$ is formed by the projections of the balanced equivalence relations on $\mathcal{G}_{1}$ that refine the lift of the input relation of $\mathcal{G}_{2}$.

(ii) The set of balanced equivalence relations on $\mathcal{G}_{1}$ that are inherited from $\mathcal{G}_{2}$ is given by the set of the lifts of the balanced equivalence relations on $\mathcal{G}_{2}$ refining the projection of the input relation for $\mathcal{G}_{1}$.

Proof (i) In symbols, we have to prove that:

$$
\Lambda_{\mathcal{G}_{2}}^{I}=\left\{\operatorname{Proj}(\bowtie): \bowtie \in \Lambda_{\mathcal{G}_{1}} \wedge \bowtie<\operatorname{Lift}\left(\sim_{I_{2}}\right)\right\}
$$

Let $\bowtie \in \Lambda_{\mathcal{G}_{1}}$ be such that $\bowtie<\operatorname{Lift}\left(\sim_{I_{2}}\right)$. Then,

$$
\operatorname{Proj}(\bowtie) \prec \operatorname{Proj}\left(\operatorname{Lift}\left(\sim_{I_{2}}\right)\right)=\sim_{I_{2}} .
$$

It follows from Lemma 4.3 (i) that $\operatorname{Proj}(\bowtie) \in \Lambda_{\mathcal{G}_{2}}$. This concludes the "つ" part. 
Suppose that $\bowtie^{\prime} \in \Lambda_{\mathcal{G}_{2}}$ and $\bowtie^{\prime}=\operatorname{Proj}(\bowtie)$ for some $\bowtie \in \Lambda_{\mathcal{G}_{1}}$. Then, $\bowtie<\sim_{I_{1}}$, so

$$
\bowtie^{\prime}=\operatorname{Proj}(\bowtie)<\operatorname{Proj}\left(\sim_{I_{1}}\right) .
$$

It follows that

$$
\operatorname{Lift}\left(\bowtie^{\prime}\right)<\operatorname{Lift}\left(\operatorname{Proj}\left(\sim_{I_{1}}\right)\right)<\sim_{I_{1}} .
$$

Thus, by Lemma 4.3 (ii), we have $\operatorname{Lift}\left(\bowtie^{\prime}\right) \in \Lambda_{\mathcal{G}_{1}}$. That is, $\bowtie \in \Lambda_{\mathcal{G}_{1}}$.

(ii) The proof is analogous.

In general, there can be balanced equivalence relations on $\mathcal{G}_{2}$ (on $\mathcal{G}_{1}$ ) that are not inherited from the balanced equivalence relations on $\mathcal{G}_{1}$ (on $\mathcal{G}_{2}$ ). We show next that the condition of the input relation on $\mathcal{G}_{2}$ to refine the projection of the input relation on $\mathcal{G}_{1}$ is sufficient to guarantee that all the balanced equivalence relations on $\mathcal{G}_{2}$ are inherited from the ones on $\mathcal{G}_{1}$.

Theorem 4.5 Let $\mathcal{G}_{2}$ be obtained from $\mathcal{G}_{1}$ by removing a cell $c$.

(i) If the input relation on $\mathcal{G}_{2}$ refines the projection of the input relation on $\mathcal{G}_{1}$, then every balanced relation on $\mathcal{G}_{2}$ is inherited from $\mathcal{G}_{1}$.

(ii) If the input relation for $\mathcal{G}_{1}$ refines the lift of the input relation on $\mathcal{G}_{2}$ then every balanced relation on $\mathcal{G}_{1}$ is inherited from $\mathcal{G}_{2}$.

Proof (i) Let $\bowtie$ be a balanced equivalence relation on $\mathcal{G}_{2}$. Consider $\tilde{\bowtie}=\operatorname{Lift}(\bowtie)$. Note that $\bowtie=\operatorname{Proj}(\tilde{\bowtie})$. Since $\bowtie$ is balanced on $\mathcal{G}_{2}$, we have $\bowtie$ refines $\sim_{I_{2}}$. Thus,

$$
\operatorname{Lift}(\bowtie)<\operatorname{Lift}\left(\sim_{I_{2}}\right)<\operatorname{Lift}\left(\operatorname{Proj}\left(\sim_{I_{1}}\right)\right)<\sim_{I_{1}}
$$

It follows from Lemma 4.3 (ii) that $\tilde{\bowtie}=\operatorname{Lift}(\bowtie) \in \Lambda_{\mathcal{G}_{1}}$ and so $\bowtie$ is a balanced relation on $\mathcal{G}_{2}$ inherited from $\mathcal{G}_{1}$.

(ii) Let $\bowtie$ be balanced on $\mathcal{G}_{1}$. Then, $\bowtie<\sim_{I_{1}}<\operatorname{Lift}\left(\sim_{I_{2}}\right)$, which implies that $[c]_{\bowtie}=\{c\}$ and $\bowtie=\operatorname{Lift}(\operatorname{Proj} \bowtie)$. Also since $\bowtie<\operatorname{Lift}\left(\sim_{I_{2}}\right)$, we have

$$
\operatorname{Proj}(\bowtie)<\operatorname{Proj}\left(\operatorname{Lift}\left(\sim_{I_{2}}\right)\right)=\sim_{I_{2}} .
$$

By Lemma 4.3, $\operatorname{Proj}(\bowtie) \in \Lambda_{\mathcal{G}_{2}}$. Therefore, $\bowtie \in \Lambda_{\mathcal{G}_{1}{ }^{\prime}}$ since $\bowtie=\operatorname{Lift}(\operatorname{Proj}(\bowtie))$.

In the next corollary we give two cases guaranteeing that the input relation on $\mathcal{G}_{2}$ refines the projection of the input relation on $\mathcal{G}_{1}$ :

Corollary 4.6 Let $\mathcal{G}_{2}$ be obtained from $\mathcal{G}_{1}$ by removing a cell c. If $\mathcal{G}_{1}$ is a regular network, or the removed cell $c$ has no outgoing edges, then every balanced relation on $\mathcal{G}_{2}$ is inherited from $\mathcal{G}_{1}$.

Proof If $\mathcal{G}_{1}$ is a regular network, then $\operatorname{Proj}\left(\sim_{I_{1}}\right)$ is the equality relation on $C_{2}$, and so $\sim_{I_{2}}$ refines $\operatorname{Proj}\left(\sim_{I_{1}}\right)$. Or if there is no outgoing edges from $c$, then $\operatorname{Proj}\left(\sim_{I_{1}}\right)=\sim_{I_{2}}$. In either case, by Theorem 4.5 , we have $\Lambda_{\mathcal{G}_{2}}=\Lambda_{\mathcal{G}_{2}}^{I}$.

Example 4.7 Consider the networks $\mathcal{G}_{1}, \mathcal{G}_{2}$ and $\mathcal{G}_{3}$ given by Figure 3, where $\mathcal{G}_{2}, \mathcal{G}_{3}$ are obtained by successively removing nodes 6 and 5 from $\mathcal{G}_{1}$. Using the algorithm 

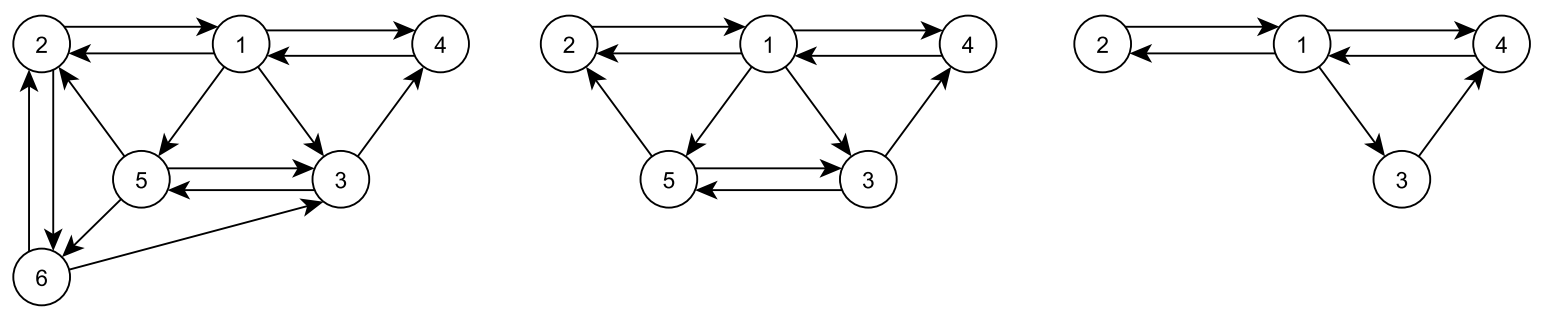

Figure 3: The network $\mathcal{G}_{2}$ is obtained from $\mathcal{G}_{1}$ by removing the cell 6; and the network $\mathcal{G}_{3}$ is obtained from $\mathcal{G}_{2}$ by removing the cell 5 .

\begin{tabular}{|l|l|l|}
\hline$\bowtie_{0}^{1}=\{\{1\},\{2\},\{3\},\{4\},\{5\},\{6\}\}$ & $\bowtie_{3}^{1}=\{\{1,4\},\{2,3\},\{5\},\{6\}\}$ & $\bowtie_{6}^{1}=\{\{1,4,5\},\{2,3\},\{6\}\}$ \\
$\bowtie_{1}^{1}=\{\{1\},\{2,3\},\{4\},\{5\},\{6\}\}$ & $\bowtie_{4}^{1}=\{\{1\},\{2,3\},\{4,5\},\{6\}\}$ & $\bowtie_{7}^{1}=\{\{1,6\},\{2,3\},\{4,5\}\}$ \\
$\bowtie_{2}^{1}=\{\{1\},\{2\},\{3\},\{4,5\},\{6\}\}$ & $\bowtie_{5}^{1}=\{\{1,6\},\{2\},\{3\},\{4,5\}\}$ & $\bowtie_{8}^{1}=\{\{1,4,5,6\},\{2,3\}\}$ \\
\hline
\end{tabular}

Table 4: The balanced relations for the network $\mathcal{G}_{1}$ of Figure 3.

presented in [2], we obtain the lattice of balanced equivalence relations on $\mathcal{G}_{1}$, as shown in Table 4.

The input equivalence relations on the $\mathcal{G}_{i}{ }^{\prime}$ s are listed below:

$$
\sim_{I_{1}}=\{\{1,4,5,6\},\{2,3\}\}, \quad \sim_{I_{2}}=\{\{1,2,3,4,5\}\}, \quad \sim_{I_{3}}=\{\{1,4\},\{2,3\}\}
$$

and their corresponding projections are given by

$$
\operatorname{Proj}\left(\sim_{I_{1}}\right)=\{\{1,4,5\},\{2,3\}\}, \quad \operatorname{Proj}\left(\sim_{I_{2}}\right)=\{\{1,2,3,4\}\}, \quad \operatorname{Proj}\left(\sim_{I_{3}}\right)=\{\{1,4\},\{2\}\} .
$$

Note that we have

$$
\sim_{I_{2}} \nless \operatorname{Proj}\left(\sim_{I_{1}}\right) \text { and } \sim_{I_{3}} \prec \operatorname{Proj}\left(\sim_{I_{2}}\right) .
$$

Consider $\mathcal{G}_{2}$ which is obtained from $\mathcal{G}_{1}$ by removing cell 6 . By Theorem 4.4 , the set of balanced equivalence relations on $\mathcal{G}_{2}$ inherited from $\mathcal{G}_{1}$ is

$\Lambda_{\mathcal{G}_{2}}^{I}=\left\{\bowtie_{0}^{2}, \bowtie_{1}^{2}, \bowtie_{3}^{2}, \bowtie_{7}^{2}, \bowtie_{8}^{2}, \bowtie_{13}^{2}\right\}=\left\{\operatorname{Proj}\left(\bowtie_{0}^{1}\right), \operatorname{Proj}\left(\bowtie_{1}^{1}\right), \operatorname{Proj}\left(\bowtie_{2}^{1}\right), \operatorname{Proj}\left(\bowtie_{3}^{1}\right), \operatorname{Proj}\left(\bowtie_{4}^{1}\right), \operatorname{Proj}\left(\bowtie_{6}^{1}\right)\right\}$.

Since $\sim_{I_{2}} \nless \operatorname{Proj}\left(\sim_{I_{1}}\right)$, there can be balanced equivalence relations on $\mathcal{G}_{2}$ that are not

\begin{tabular}{|c|c|c|c|}
\hline$\bowtie_{0}^{2}=\{\{1\},\{2\},\{3\},\{4\},\{5\}\}$ & $\bowtie_{4}^{2}=\{\{1\},\{2,3,5\},\{4\}\}$ & $\bowtie_{8}^{2}=\{\{1\},\{2,3\},\{4,5\}\}$ & $\bowtie_{12}^{2}=\{\{1,2,3\},\{4,5\}\}$ \\
\hline$\bowtie_{1}^{2}=\{\{1\},\{2,3\},\{4\},\{5\}\}$ & $\bowtie_{5}^{2}=\{\{1\},\{2\},\{3,4,5\}\}$ & $\bowtie_{9}^{2}=\{\{1\},\{2,4\},\{3,5\}\}$ & $\bowtie_{13}^{2}=\{\{1,4,5\},\{2,3\}\}$ \\
\hline$\bowtie_{2}^{2}=\{\{1\},\{2\},\{3,5\},\{4\}\}$ & $\bowtie_{6}^{2}=\{\{1,2\},\{3\},\{4,5\}\}$ & $\bowtie_{10}^{2}=\{\{1,2\},\{3,4,5\}\}$ & $\bowtie_{14}^{2}=\{\{1\},\{2,3,4,5\}\}$ \\
\hline$\bowtie_{3}^{2}=\{\{1\},\{2\},\{3\},\{4,5\}\}$ & $\triangle_{7}^{2}=\{\{1,4\},\{2,3\},\{5\}\}$ & $\triangle_{11}^{2}=\{\{1,4\},\{2,3,5\}\}$ & $\bowtie_{15}^{2}=\{\{1,2,3,4,5\}\}$ \\
\hline
\end{tabular}
inherited from $\mathcal{G}_{1}$. The total list of balanced equivalence relations on $\mathcal{G}_{2}$ is given in Table 5.

Table 5: The balanced relations for the network $\mathcal{G}_{2}$ of Figure 3. 
Next, consider the network $\mathcal{G}_{3}$ which is obtained from $\mathcal{G}_{2}$ by removing the cell 5 . By Theorem 4.5, since $\sim_{I_{3}} \prec \operatorname{Proj}\left(\sim_{I_{2}}\right)$, we have

$$
\Lambda_{\mathcal{G}_{3}}=\Lambda_{\mathcal{G}_{3}}^{I}=\left\{\bowtie_{0}^{3}, \bowtie_{1}^{3}, \bowtie_{2}^{3}\right\}=\left\{\operatorname{Proj}\left(\bowtie_{0}^{2}\right), \operatorname{Proj}\left(\bowtie_{1}^{2}\right), \operatorname{Proj}\left(\bowtie_{7}^{2}\right)\right\}
$$

The list of the balanced equivalence relations on $\mathcal{G}_{3}$ appears in Table 6 .

$$
\bowtie_{0}^{3}=\{\{1\},\{2\},\{3\},\{4\}\} \quad \bowtie_{1}^{3}=\{\{1\},\{2,3\},\{4\}\} \quad \star_{2}^{3}=\{\{1,4\},\{2,3\}\}
$$

Table 6: The balanced equivalence relations for the network $\mathcal{G}_{3}$ of Figure 3.

\section{Acknowledgments}

The authors thank the referees for their comments that much improved the way the main results of this paper are stated. HR thanks the University of Porto for its hospitality and acknowledges additional support from the European Regional Development Fund through the programme COMPETE and by the Portuguese Government through the FCT - Fundação para a Ciência e a Tecnologia under the project PTDC/MAT/100055/2008.
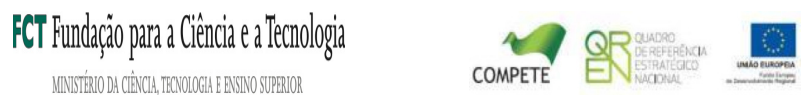

\section{References}

[1] M. Aguiar, P. Ashwin, A. Dias, and M. Field. Dynamics of coupled cell networks: synchrony, heteroclinic cycles and inflation. J. Nonlinear Sci. 21 (2) (2011) 271-323.

[2] M.A.D. Aguiar and A.P.S. Dias. The Lattice of Synchrony Subspaces of a Coupled Cell Network: Characterization and Computation Algorithm. J. Nonlinear Sci. 24 (6) (2014) 949-996.

[3] M.A.D. Aguiar and A.P.S. Dias. Regular Synchrony Lattices of Product Coupled Cell Networks. Chaos 25013108 (2015) 1-10. DOI: 10.1063/1.4905703

[4] M.A.D. Aguiar and H. Ruan. Evolution of Synchrony under Combination of Coupled Cell Networks. Nonlinearity 25 (2012) 3155-3187.

[5] J. W. Aldis. A polynomial time algorithm to determine maximal balanced equivalence relations. J. Bifur. Chaos Appl. Sci. Engrg. 18 (2) (2008) 407-427.

[6] F. M. Atay and T. Biyikoglu. Graph operations and synchronization of complex networks. Physical Review E 72 (2005) 016217.

[7] G. Chen and Z. Duan. Network synchronizability analysis: A graph-theoretic approach. Chaos $\mathbf{1 8}$ (2008) 037102.

[8] M. Field. Combinatorial dynamics. Dyn. Syst. 19 (3) (2004) 217-243. 
[9] M. Golubitsky and I. Stewart. Nonlinear dynamics of networks: the groupoid formalism. Bull. Amer. Math. Soc. 43 (3) (2006) 305-364.

[10] M. Golubitsky, I. Stewart and A. Török. Patterns of Synchrony in Coupled Cell Networks with Multiple Arrows. SIAM J. Appl. Dynam. Sys. 4 (1) (2005) 78-100.

[11] A. Hagberg and D.A. Schult. Rewiring networks for synchronization. Chaos 18037105 (2008) 1-7.

[12] W. Lu, F. M. Atay and J. Jost. Synchronization of Discrete-Time Dynamical Networks with TimeVarying Couplings. SIAM J. Math. Anal. 39 (4) (2007) 1231-1259.

[13] N. M. Luscombe, M. M. Babu, H. Yu, M. Snyder, S. A. Teichmann, M. Gerstein. Genomic analysis of regulatory network dynamics reveals large topological changes. Nature 431 (2004) 308-312. doi:10.1038/nature02782

[14] I. Stewart. The lattice of balanced equivalence relations of a coupled cell network. Math. Proc. Cambridge Philos. Soc. 143 (1) (2007) 165-183.

[15] I. Stewart, M. Golubitsky and M. Pivato. Symmetry groupoids and patterns of synchrony in coupled cell networks. SIAM J. Appl. Dynam. Sys. 2 (4) (2003) 609-646.

[16] B. Zhang, H. Li, R. B. Riggins, M. Zhan, J. Xuan, Z. Zhang, E. P. Hoffman, R. Clarke and Y. Wang. Differential dependency network analysis to identify condition-specific topological changes in biological networks. Bioinformatics 25 (4) (2009) 526-532. doi: 10.1093/bioinformatics/btn660 